\title{
Intracranial chordoma: radiosurgery, hypofractionated stereotactic radiotherapy and treatment outcomes
}

\author{
Aleksandra Napieralska, Sławomir Blamek \\ Radiotherapy Department, Maria Sklodowska-Curie National Research Institute of Oncology Gliwice Branch, Gliwice, Poland
}

\begin{abstract}
Background: The aim of the study was to assess the results of stereotactic radiosurgery and hypofractionated stereotactic radiotherapy (SRS/SRT) for skull base chordomas.

Materials and methods: Twenty-three patients aged 12-75 were treated with SRS/SRT due to skull base chordoma. In 19 patients SRS/SRT was a part of the primary therapy, while in 4, a part of the treatment of recurrence. In 4 patients SRS/SRT was used as a boost after conventional radiotherapy and in 19 cases it was the only irradiation method applied. Patients were irradiated to total dose of 6-35 Gy and median total equivalent dose of $52 \mathrm{~Gy}$.

Results: During median follow-up of 39 months, 4 patients died. One-, two- and five-year OS was 95\%, 89\% and 69\%, respectively. In nine patients, progression of the disease was diagnosed during study period. One-, two- and five-year progression free survival (PFS) from the end of radiotherapy was $81 \%, 59 \%$ and $43 \%$, respectively. Radiotherapy was well tolerated and only two patients in our group experienced moderate treatment-related toxicity.

Conclusion: SRS/SRT alone or in combination with surgery is a safe and effective method of irradiation of patients with skull base chordomas. High $\mathrm{EQD}_{2}$ is necessary to achieve satisfactory treatment results.
\end{abstract}

Key words: chordoma; rare tumors; radiotherapy; stereotactic radiotherapy; skull base tumors

Rep Pract Oncol Radiother 2021;26(5):764-772

\section{Introduction}

Chordomas (WHO Grade I) are rare tumors which account for $0.1-0.2 \%$ of all primary intracranial tumors [1]. They are described as slow growing and locally aggressive tumors with high tendency to local relapse and low incidence of distant metastases [1-3]. Only 25 to $40 \%$ of them are located at the base of the skull in the proximity of the clivus, while over half of them (50-60\%) appear in the sacrum and the rest in the upper part of the cervical spine (15\%) [1-6]. Most of the patients are diagnosed at age of 40 to 50 with slight predominance in men
$[1,2]$. The proximity of critical organs. such as the brain stem, optic chiasm or large intracranial vessels combined with a high rate of local recurrence, makes the treatment of patients with chordoma very difficult $[2,4,6-8]$.

Due to rarity of chordomas, there are no phase III randomized clinical trials and only a few phase II trials. Currently, approved therapeutic management of patients with chordomas is based on the experience presented in retrospective reports. In most centers, surgery is the standard of the primary and recurrence treatment $[3,5,6]$. Radical (R0) surgery is usually very difficult technically and

Address for correspondence: Aleksandra Napieralska, Radiotherapy Department, Maria Sklodowska-Curie National Research Institute of Oncology Gliwice Branch, ul. Wybrzeże AK 15, Gliwice 44-100, Poland, tel: (+48) 3227880 01; e-mail: olanapieralska@gmail.com, aleksandra.napieralska@io.gliwice.pl

This article is available in open access under Creative Common Attribution-Non-Commercial-No Derivatives 4.0 International (CC BY-NC-ND 4.0) license, allowing to download articles and share them with others as long as they credit the authors and the publisher, but without permission to change them in any way or use them commercially 
until recent years was associated with significant mortality. The development of endoscopic surgery, neuronavigation and neuroimaging have contributed to improve the treatment results in this area $[2,3,5,6]$.

Radiotherapy (RT) is used after primary surgical treatment as a part of the recurrence therapy and is also the treatment of choice in cases considered to be unresectable [2, 5, 7-9]. Apart from the standard radiotherapy techniques, there is an increasing number of patients who received stereotactic radiosurgery (SRS), hypofractionated stereotactic radiotherapy (SRT) or proton therapy [2, 4, 5, 7-9]. These techniques allow high and homogenic radiation dose to be delivered to target volume (tumor or tumor bed) with relatively low doses to nearby organs $[8,9]$.

The aim of the study was to assess the outcome of patients who received stereotactic radiosurgery or hypofractionated stereotactic radiotherapy due to skull or cervical spine chordomas.

\section{Materials and methods}

All patients who received radiotherapy between years 2001 and 2017 due to chordoma were identified. Among them, 23 consecutive patients (12 males, 11 females) aged 12-75 years (median 53 years) had chordoma located in the skull or cervical spine and were enrolled into the study. All of them received stereotactic radiosurgery (SRS) or hypofractionated stereotactic radiotherapy (SRT). Irradiation techniques included: 3D CRT (2-12 treatment fields) in 6 patients, intensity modulated radiation therapy (IMRT, 6-10 treatment fields) in 8 patients and volumetric modulated arc therapy (VMAT) with two arcs in one patient. Eight patients were irradiated with the CyberKnife system. Data concerning treatment results and irradiation details was collected retrospectively from medical records and treatment plans.

All patients had a good performance status (39\% ECOG 0, 61\% ECOG 1). The symptoms occurred in all patients 1 to 24 months before diagnosis (mean $7)$. The most common were: headaches (52\%), visual disturbances (43\%) and symptoms caused by damage or pressure of cranial nerves (61\%).The diagnosis of chordoma was based in 21 cases (91\%) on histopathological examination of the tumor tissue samples obtained during surgery. In the other two patients (patients who refused to have surgery) the diagnosis was based on a characteristic radiological image of the tumor.

The majority of patients had tumor located in the skull $(83 \%)$. The others had infiltration of the cervical spine (on the level of $\mathrm{C} 2-\mathrm{C} 4$ ). In 8 patients the tumor was located in direct proximity of the cerebral vessels. Most of the chordomas infiltrated the sphenoid bone (69\%), part of them, the temporal bone (22\%), occipital bone (17\%) and in 1 case the infiltration of the dura mater was described. In majority of the patients tumor compressed the neighboring structures - in 8 patients, the brain stem, in 6 , the pituitary gland and in 3 , the optic chiasm. Median dimensions of chordoma assessed on radiological preoperative imaging were $34 \times 26$ x $30 \mathrm{~mm}$ (range: 8-66). Median preoperative tumor volume was $30 \mathrm{cc}$ (mean 44; range: $2.5-201 \mathrm{cc}$ ).

In all patients the irradiated volume consisted of tumor itself (GTV - gross tumor volume) or/with tumor bed (in patients after radical surgery; CTV - clinical target volume) with additional margin which accounts for intra- and infrafraction motion (PTV - planned target volume).

Response to the treatment was evaluated with a comparative analysis of diagnostic images (magnetic resonance imaging - MRI) taken before and after irradiation and classified as: stagnation (stable size of the tumor), regression (reduction of the size of tumor) or progression (increase in the size of the tumor).

In statistical analysis Kaplan-Meier method was used to calculate overall survival (OS) and progression free survival (PFS). In order to evaluate the impact of biological and physical factors on OS and PFS, the log rank test was used. Factors included into analysis were: sex, date of primary diagnosis, primary tumor characteristics and site (spine/skull), type of treatment received, indication for RT (primary treatment, boost, management of a recurrence), performance status during $\mathrm{RT}$, irradiated volume, age, duration of symptoms, radiotherapy technique, time between surgery and irradiation, recurrence after primary surgical treatment, total and fraction dose. Follow-up was calculated from the date of the end of SRS/SRT to the date of death or last contact. P-value of less than 0.05 was considered statistically significant. EORTC/ RTOG toxicity criteria were used to assess treatment morbidity [10]. 


\section{Results}

Surgery was the primary treatment in nineteen patients. The type of resection was evaluated based on neurosurgeon assessment during the surgery and with diagnostic imaging performed after the surgery, but only $22 \%$ of patients had radical surgery, and all the others had subtotal resection of the tumor. Relapse was observed after surgery in 4 patients. All of them underwent another surgery (three of them twice). Median time between surgery and radiotherapy was 5 months (range: 2-32).
Stereotactic radiotherapy/radiosurgery was a single radiation treatment modality in 19 patients, in 4 was used as a boost to conventionally fractionated radiotherapy (fraction dose of 1.8-3.0 Gy and total dose ranging between 27 and $60 \mathrm{~Gy}$ ). Total and fraction doses used in patients treatment are presented in Table 1.

In 19 patients SRS/SRT was a part of the primary therapy (in 15 as an adjuvant treatment after surgery and in 4 it was the only treatment method applied). In 4 patients SRS/SRT was a part of the treatment of recurrence. In 4 patients SRS/SRT was used as

Table 1. Treatment details specified for individual patients

\begin{tabular}{|c|c|c|c|c|c|c|c|}
\hline Patient & Site & Surgery & $\begin{array}{c}\text { RT } \\
\text { technique }\end{array}$ & $\begin{array}{l}\text { Fraction } \\
\text { dose (Gy) }\end{array}$ & $\begin{array}{c}\text { Total } \\
\text { dose (Gy) }\end{array}$ & $\begin{array}{c}E Q D 2 \\
(\alpha / \beta=3)\end{array}$ & Treatment results \\
\hline $\begin{array}{l}1 \\
F, 53 y\end{array}$ & Spine, C4 & Non-radical & SRT & 5 & 30 & 40.0 & $\begin{array}{c}\text { Recurrence, } \\
\text { PFS } 32 \text { months, alive }\end{array}$ \\
\hline $\begin{array}{l}2 \\
F, 61 y \\
\end{array}$ & Spine, C2 & $\begin{array}{l}\text { Radical, } \\
\text { recurrence }\end{array}$ & $\mathrm{XRT}+\mathrm{SRS}$ & $1.8+6$ & $43.2+6$ & 52.3 & $\begin{array}{l}\text { Recurrence, metastases, } \\
\text { PFS } 22 \text { months, alive }\end{array}$ \\
\hline $\begin{array}{l}3 \\
F, 13 y\end{array}$ & Spine, C3 & Non-radical & SRS & 9 & 9 & 21.6 & Recurrence, PFS 12 months, alive \\
\hline $\begin{array}{l}4 \\
F, 27 y\end{array}$ & Spine, C2 & $\begin{array}{l}\text { Non-radical, } \\
\text { recurrence }\end{array}$ & SRT & 5 & 25 & 40 & Alive \\
\hline $\begin{array}{l}5 \\
M, 54 y\end{array}$ & $\begin{array}{l}\text { Skull. Sphenoid } \\
\text { bone }\end{array}$ & Non-radical & SRT & 8 & 32 & 70.4 & $\begin{array}{l}\text { Free from disease, } \\
\text { FU } 17 \text { months, alive }\end{array}$ \\
\hline $\begin{array}{l}6 \\
M, 37 y\end{array}$ & $\begin{array}{l}\text { Skull. Sphenoid } \\
\text { bone }\end{array}$ & Radical & SRS & 18 & 18 & 75.6 & $\begin{array}{l}\text { Recurrence, PFS } 7 \text { months, } \\
\text { OS } 49 \text { months }\end{array}$ \\
\hline $\begin{array}{l}7 \\
F, 69 y\end{array}$ & $\begin{array}{l}\text { Skull. Sphenoid } \\
\text { bone }\end{array}$ & Non-radical & SRT & 8 & 24 & 52.8 & $\begin{array}{c}\text { Free from disease, FU } 6 \text { months, } \\
\text { alive }\end{array}$ \\
\hline $\begin{array}{l}9 \\
F, 46 y \\
\end{array}$ & $\begin{array}{l}\text { Skull. Sphenoid } \\
\text { bone }\end{array}$ & Radical & SRS & 10 & 10 & 26 & Recurrence, PFS 85 months, alive \\
\hline $\begin{array}{l}10 \\
M, 57 y\end{array}$ & $\begin{array}{l}\text { Skull. Sphenoid } \\
\text { bone }\end{array}$ & - & SRT & 6 & 24 & 43.2 & $\begin{array}{c}\text { Free from disease, FU } 42 \text { months, } \\
\text { alive }\end{array}$ \\
\hline $\begin{array}{l}11 \\
F, 63 y\end{array}$ & $\begin{array}{l}\text { Skull. Sphenoid } \\
\text { bone }\end{array}$ & Non-radical & SRT & 6 & 18 & 32.4 & $\begin{array}{l}\text { Free from disease, OS } 12 \text { months, } \\
\text { dead due to breast cancer }\end{array}$ \\
\hline $\begin{array}{l}12 \\
M, 64 y\end{array}$ & $\begin{array}{l}\text { Skull. Sphenoid } \\
\text { bone }\end{array}$ & Non-radical & SRT & 8 & 32 & 70.4 & Alive \\
\hline $\begin{array}{l}13 \\
M, 56 y\end{array}$ & $\begin{array}{l}\text { Skull. Sphenoid } \\
\text { bone }\end{array}$ & - & SRT & 8 & 16 & 35.2 & $\begin{array}{l}\text { Progression, metastases, } \\
\text { PFS } 8 \text { months, OS } 46 \text { months }\end{array}$ \\
\hline $\begin{array}{l}14 \\
M, 56 y\end{array}$ & $\begin{array}{l}\text { Skull. Sphenoid } \\
\text { bone }\end{array}$ & Non-radical & SRT & 7 & 35 & 70 & $\begin{array}{c}\text { Free from disease, FU } 39 \text { months, } \\
\text { alive }\end{array}$ \\
\hline $\begin{array}{l}15 \\
F, 48 y\end{array}$ & $\begin{array}{l}\text { Skull. Sphenoid } \\
\text { bone }\end{array}$ & - & $\mathrm{XRT}+\mathrm{SRT}$ & $3+3$ & $27+12$ & 46.8 & Progression, PFS 20 months, alive \\
\hline $\begin{array}{l}16 \\
M, 23 y\end{array}$ & $\begin{array}{l}\text { Skull. Sphenoid } \\
\text { bone }\end{array}$ & Non-radical & SRS & 14 & 14 & 47.6 & Free from disease, OS 3 months \\
\hline $\begin{array}{l}17 \\
F, 65 y\end{array}$ & $\begin{array}{l}\text { Skull. Sphenoid } \\
\text { bone }\end{array}$ & Non-radical & $\mathrm{XRT}+\mathrm{SRT}$ & $2+6$ & $54+12$ & 75.6 & $\begin{array}{c}\text { Free from disease, FU } 35 \text { months, } \\
\text { alive }\end{array}$ \\
\hline $\begin{array}{l}18 \\
M, 66 y\end{array}$ & $\begin{array}{l}\text { Skull. Sphenoid } \\
\text { bone }\end{array}$ & - & SRT & 8 & 32 & 70.4 & Progression, PFS 47 months, alive \\
\hline $\begin{array}{l}19 \\
M, 64 y\end{array}$ & $\begin{array}{l}\text { Skull. Sphenoid } \\
\text { bone }\end{array}$ & Radical & SRT & 6 & 18 & 32.4 & $\begin{array}{c}\text { Free from disease, FU } 53 \text { months, } \\
\text { alive }\end{array}$ \\
\hline
\end{tabular}


Table 1. Treatment details specified for individual patients

\begin{tabular}{|c|c|c|c|c|c|c|c|}
\hline Patient & Site & Surgery & $\begin{array}{c}\text { RT } \\
\text { technique }\end{array}$ & $\begin{array}{l}\text { Fraction } \\
\text { dose (Gy) }\end{array}$ & $\begin{array}{c}\text { Total } \\
\text { dose (Gy) }\end{array}$ & $\begin{array}{c}\text { EQD2 } \\
(\alpha / \beta=3)\end{array}$ & Treatment results \\
\hline $\begin{array}{l}20 \\
M, 45 y\end{array}$ & $\begin{array}{l}\text { Skull. Sphenoid } \\
\text { bone }\end{array}$ & Non-radical & $\mathrm{XRT}+\mathrm{SRT}$ & $1.8+9$ & $45+18$ & 86.4 & $\begin{array}{c}\text { Free from disease, FU } 33 \text { months, } \\
\text { alive }\end{array}$ \\
\hline $\begin{array}{l}21 \\
M, 24 y\end{array}$ & $\begin{array}{l}\text { Skull. Sphenoid } \\
\text { bone }\end{array}$ & Radical & SRT & 7 & 28 & 56 & $\begin{array}{c}\text { Free from disease, FU } 79 \text { months, } \\
\text { alive }\end{array}$ \\
\hline $\begin{array}{l}22 \\
M, 52 y\end{array}$ & $\begin{array}{l}\text { Skull. Sphenoid } \\
\text { bone }\end{array}$ & Non-radical & $\mathrm{XRT}+\mathrm{SRT}$ & $2+6$ & $60+12$ & 81.6 & $\begin{array}{l}\text { Free from disease, FU } 110 \text { months, } \\
\text { alive }\end{array}$ \\
\hline $\begin{array}{l}23 \\
F, 23 y\end{array}$ & $\begin{array}{l}\text { Skull. Sphenoid } \\
\text { bone }\end{array}$ & $\begin{array}{l}\text { Non-radical, } \\
\text { recurrence }\end{array}$ & SRT & 9 & 18 & 43.2 & $\begin{array}{l}\text { Free from disease, FU } 22 \text { months, } \\
\text { alive }\end{array}$ \\
\hline $\begin{array}{l}24 \\
F, 76 y\end{array}$ & $\begin{array}{l}\text { Skull. Sphenoid } \\
\text { bone }\end{array}$ & $\begin{array}{l}\text { Non-radical, } \\
\text { recurrence }\end{array}$ & SRT & 8 & 32 & 70.4 & Progression, PFS 12 months, alive \\
\hline
\end{tabular}

*EQD2 - biological equivalent dose; F-female; FU — follow-up; M- male; OS — overall survival; PFS — progression free survival; RT — radiotherapy;

SRS — stereotactic radiosurgery; SRT — stereotactic radiotherapy; XRT — conventional fractionated radiotherapy; $y$-years

a boost after conventional radiotherapy (with fraction dose of 1.8-3.0Gy and total dose of 27-60 Gy) and in 19 cases it was the only irradiation method applied. Patients were irradiated with 1 to 5 fractions of 3 to 18 Gy to total dose of 6 to 35 Gy and the total equivalent 2 Gy dose (EQD2 ${ }_{3}$, calculated assuming $\alpha / \beta$ of 3 for chordoma) after adding dose given with conventional irradiation ranged from 21.6 to 86.4 Gy (median 52 Gy). Median volume of PTV was 17 cc (mean 55; range: $1.4-463.8 \mathrm{cc}$ ). Radiotherapy was well tolerated and only two patients in our group experienced moderate treatment-related toxicity. In both of them diminished hormone secretion by the pituitary gland was diagnosed. Both of them required hormone supplementation and are currently under the care of an endocrinologist.

During median follow-up of 39 months (range: 0-141 months) 4 patients died. One-, two- and five-year OS was $95 \%, 89 \%$ and $69 \%$, respectively (Fig. 1). Twenty patients had regular visits after the treatment (three did not show up for the planned visit). None of analyzed factors had a statistically significant influence on OS. All patients with chordoma located in the cervical spine are alive.

In nine patients progression of the disease was diagnosed during the study period. Four of them had a salvage surgery (one of them twice) and three of them were irradiated (previously received total equivalent dose was 35.2 Gy, 52.3Gy, and 70.4 Gy). One of them (the one who received $35.2 \mathrm{~Gy}$ ) had SRS twice, and the doses applied were 7 Gy in one fraction $\left(\mathrm{EQD} 2_{3}=14 \mathrm{~Gy}\right)$ and $8 \mathrm{~Gy}$ in one fraction $\left(\mathrm{EQD} 2_{3}=17.6 \mathrm{~Gy}\right)$. Total EQD2 ${ }_{3}$ delivered in that patient was 66.8 Gy. Despite this treatment this patient died due to progression of the disease and

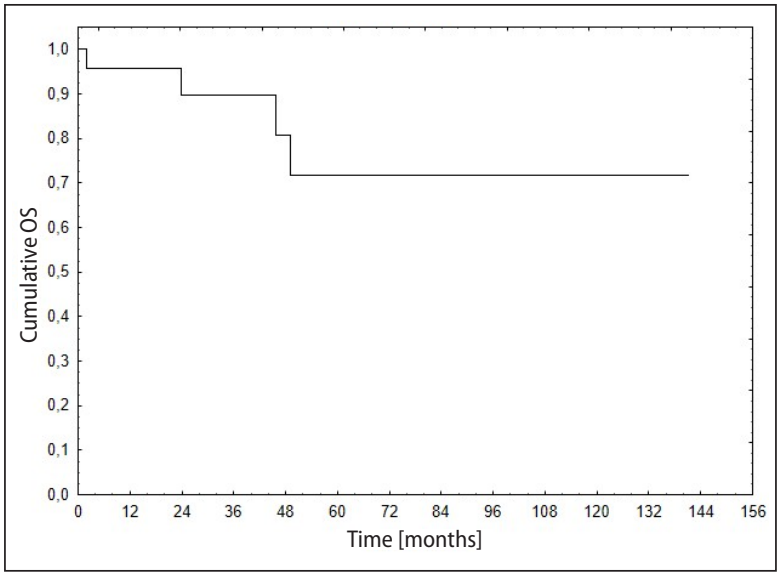

Figure 1. Overall survival

distant metastases. In the case of the second of the reirradiated patients (who received 52.3 Gy), conventional radiotherapy was applied for treatment of the recurrence, with fraction dose of 2 Gy to total dose of $30 \mathrm{~Gy}$ (total equivalent 2 Gy dose from two RT treatments was $82.3 \mathrm{~Gy}$ ). After radiotherapy this patient underwent another two salvage surgeries for recurrence of the tumor and in the recent MRI another recurrence and metastases to the mandible and cervical part of the spine were diagnosed. He refused further treatment and remains under observation. Beside the two mentioned above, distant metastases were found in another patient who was referred to symptomatic treatment and died two years after the diagnosis of metastases. The third of the reirradiated patients received $25 \mathrm{~Gy}$ in $5 \mathrm{~Gy}$ fractions (EQD2 ${ }_{3}$ of $40 \mathrm{~Gy}$ ) and is alive with no signs of disease 20 months after the second course of radiotherapy. 


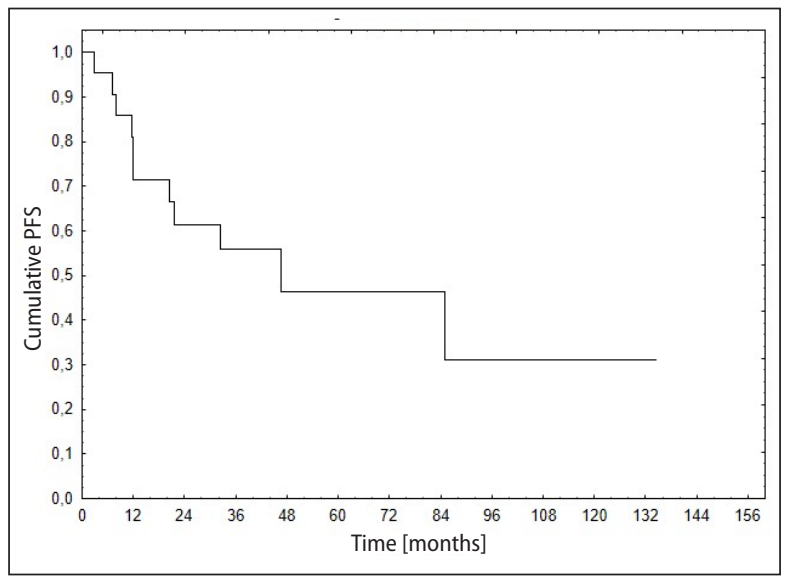

Figure 2. Progression free survival

One-, two- and five-year progression free survival (PFS) calculated from the end of radiotherapy was $81 \%, 59 \%$ and $43 \%$, respectively (Fig. 2, Tab. 1).

\section{Discussion}

The reported number of patients who received adjuvant radiotherapy varies between authors and ranges between 21 and $100 \%[1,2-5,7,9,11-13$,
$26,39,40]$. Radiotherapy is used not only as adjuvant therapy after surgery but also as a treatment of choice for patients with inoperable tumors [25, $30,35,39,40]$. Five-year overall survival (OS) and five-year progression free survival (PFS) of patients irradiated with various RT techniques is presented in Tables 2 and 3.

Also in our group in 4 patients who did not undergo surgery, irradiation was the only treatment method applied. The doses used in SRS/SRT vary in the literature and range from 9 to $68.4 \mathrm{~Gy}$ (Tab. 2 and 3). The large range of total 2 Gy equivalent doses (and hence, fraction and total doses) in our group resulted from a retrospective nature of the analysis and lack of established protocols of treatment of patients with chordomas. The treatment was individualized taking into account the volume of the tumor, its localization and prior use of radiation. Despite this, we did not observe a clear correlation between the dose delivered and treatment effect (the difference was statistically insignificant). Some researchers found correlation between the applied total dose and OS and local control - Koga et al. reported a statistically significant difference between patients treated with

Table 2. Five-year overall survival and five-year progression free survival according to applied radiotherapy technique

\begin{tabular}{|c|c|c|c|c|c|}
\hline Study & $\begin{array}{l}\text { Number } \\
\text { of patients }\end{array}$ & Radiotherapy & 5-year OS & $\begin{array}{l}\text { 5-year } \\
\text { PFS }\end{array}$ & $\begin{array}{l}\text { Mean follow-up } \\
\text { [months] }\end{array}$ \\
\hline $\begin{array}{l}\text { Ito et al. } \\
(2010)\end{array}$ & 15 & $13 \%-\mathrm{XRT} ; \mathrm{GK}$ at recurrence only & $100 \%$ & $48 \%$ & 82 \\
\hline $\begin{array}{l}\text { Takahashi et al. } \\
\text { (2009) }\end{array}$ & 32 & $\begin{array}{c}100 \% \text { - all with residual tumor; } \\
\text { carbon ions: } 9 / 32 ; \text { XRT: 7/32; PBRT: 5/32 }\end{array}$ & $92 \%$ & $29 \%$ & 36 \\
\hline $\begin{array}{l}\text { Schultz-Ertner et al. } \\
(2007)\end{array}$ & 96 & $\begin{array}{l}100 \% \text { - carbon ions for patients } \\
\text { with postoperative residual tumor }\end{array}$ & $88 \%$ & $70 \%$ & 31 \\
\hline $\begin{array}{l}\text { Yoneoka et al. } \\
(2008)\end{array}$ & 13 & $\begin{array}{c}100 \%-\text { XRT }(10 / 13), \text { GK }(3 / 13) ; \\
\text { adjuvant RT in all patients (primary XRT) }\end{array}$ & $82 \%$ & $69 \%$ & 122 \\
\hline $\begin{array}{l}\text { Debus et al. } \\
(2000)\end{array}$ & 37 & fXRT for residual tumor & $82 \%$ & $50 \%$ & 27 \\
\hline $\begin{array}{l}\text { Tzortzidis et al. } \\
\text { (2006) }\end{array}$ & 74 & $\begin{array}{l}71.6 \% \text { - PBRT (38\%), GK (43\%), XRT (19\%); } \\
\text { RT withheld for gross-total resections }\end{array}$ & $\begin{array}{l}82 \% \\
\text { (last control) }\end{array}$ & $41 \%$ & 96 \\
\hline $\begin{array}{l}\text { Noël et al. } \\
(2005)\end{array}$ & 100 & $70 \%$ - combination of XRT and PBRT & $80 \%$ & $\begin{array}{c}54 \% \\
\text { (4 years) }\end{array}$ & 31 \\
\hline $\begin{array}{l}\text { Cho et al. } \\
(2008)\end{array}$ & 16 & $\begin{array}{l}63 \% \mathrm{XRT} \text {, standard adjuvant } \mathrm{XRT} ; \\
\text { GK for residual tumor or recurrence }\end{array}$ & $80 \%$ & $40 \%$ & 56 \\
\hline $\begin{array}{l}\text { Hug et al. } \\
\text { (1999) }\end{array}$ & 33 & $100 \%$ - PBRT; primary for residual tumor & $79 \%$ & $59 \%$ & 33 \\
\hline $\begin{array}{l}\text { Pamir et al. } \\
(2004)\end{array}$ & 26 & $\begin{array}{l}27 \%-\text { GK; for residual tumor }<30 \mathrm{~cm}^{3} \text {; } \\
\text { RT withheld for gross-total resections }\end{array}$ & $77 \%$ & $46 \%$ & 49 \\
\hline $\begin{array}{l}\text { Almefty et al. } \\
(2007)\end{array}$ & 89 & $75 \%$ PBRT & $75 \%$ & $51 \%$ & 48 \\
\hline
\end{tabular}


Table 2. Five-year overall survival and five-year progression free survival according to applied radiotherapy technique

\begin{tabular}{|c|c|c|c|c|c|}
\hline Study & $\begin{array}{l}\text { Number } \\
\text { of patients }\end{array}$ & Radiotherapy & 5-year OS & $\begin{array}{l}\text { 5-year } \\
\text { PFS }\end{array}$ & $\begin{array}{l}\text { Mean follow-up } \\
\text { [months] }\end{array}$ \\
\hline $\begin{array}{l}\text { Sen et al. } \\
(2010)\end{array}$ & 71 & $\begin{array}{l}42 \% \text { - PBRT primary, RT only for postoperative } \\
\text { residual tumor }\end{array}$ & $75 \%$ & $64 \%$ & 66 \\
\hline $\begin{array}{l}\text { lgaki et al. } \\
\text { (2004) }\end{array}$ & 13 & $100 \%$ - PBRT, only for postoperative residual tumor & $67 \%$ & $42 \%$ & 69 \\
\hline $\begin{array}{l}\text { Sami et al. } \\
\text { (2007) }\end{array}$ & 49 & $39 \%-\mathrm{XRT}$ & $65 \%$ & $15 \%$ & 63 \\
\hline $\begin{array}{l}\text { Tamaki et al. } \\
(2001)\end{array}$ & 17 & $\begin{array}{l}\text { 76\%; XRT for residual tumor, including neartotal } \\
\text { resection (no RT for gross resection) }\end{array}$ & $64 \%$ & $51 \%$ & 59 \\
\hline $\begin{array}{l}\text { Foweraker et al. } \\
(2007)\end{array}$ & 9 & $100 \%-\mathrm{XRT}$ for all patients & $63 \%$ & $80 \%$ & 38 \\
\hline $\begin{array}{l}\text { Pallini et al. } \\
\text { (2003) }\end{array}$ & 22 & $58 \%$ - RT, PBRT; for residual tumor only & $61 \%$ & $50 \%$ & 63 \\
\hline $\begin{array}{l}\text { Stüer et al. } \\
\text { (2006) }\end{array}$ & 11 & $\begin{array}{c}\text { 100\% - all postoperative RT: PBRT: } 2 / 11 \\
\text { carbon: } 4 / 11 \text {; PBRT/carbon: } 1 / 11 ; \text { GK: } 1 / 11 \text {; XRT: } 1 / 11\end{array}$ & N/A & $51 \%$ & 36 \\
\hline
\end{tabular}

GK — Gamma Knife radiosurgery; N/A — data non available; OS — overall survival; PBRT — proton radiotherapy; PFS — progression free survival; $\mathrm{RT}$ — radiotherapy; XRT — conventional fractionated radiotherapy

Table 3. Five-year overall survival and five-year progression free survival according to applied stereotactic radiosurgery technique

\begin{tabular}{|c|c|c|c|c|c|c|}
\hline Study & $\begin{array}{l}\text { Number } \\
\text { of patients }\end{array}$ & SRS/ SRT/ RT & 5-year OS & $\begin{array}{l}\text { 5-year } \\
\text { PFS }\end{array}$ & $\begin{array}{l}\text { Follow-up } \\
\text { [months] }\end{array}$ & Complications \\
\hline $\begin{array}{l}\text { Muthukumar et al. } \\
\text { (1998) }\end{array}$ & 15 (9 ch) & $\begin{array}{l}\text { GK, dose: } 12-20 \text { Gy } \\
\text { (median 18) in } 1 \text { fraction }\end{array}$ & $\mathrm{N} / \mathrm{A}$ & $66 \%$ & 48 (mean) & None \\
\hline $\begin{array}{l}\text { Chang et al. } \\
(2001)\end{array}$ & 10 & $\begin{array}{l}5 \text { CK, } 5 \text { XRT, dose: } 18-24 \text { Gy } \\
\text { (mean 19.4) in } 1 \text { fraction }\end{array}$ & $\begin{array}{l}\text { 4-year: } \\
\text { 100\% }\end{array}$ & $\begin{array}{l}\text { 4-year: } \\
80 \%\end{array}$ & 48 (mean) & No radionecrosis \\
\hline $\begin{array}{l}\text { Gwak et al. } \\
(2005)\end{array}$ & $\begin{array}{c}9 \\
(7 \mathrm{ch})\end{array}$ & $\begin{array}{c}\text { CK, dose: } 21-43.6 \mathrm{~Gy} \\
\text { (median 35) in 3-5 fraction }\end{array}$ & $\begin{array}{l}\text { 2-year: } \\
\text { 100\% }\end{array}$ & $\begin{array}{c}\text { 2-year: } \\
86 \%\end{array}$ & 24 (median) & $\begin{array}{l}2 \text { RT induced } \\
\text { myelopathy }\end{array}$ \\
\hline $\begin{array}{l}\text { Krishnan et al. } \\
(2005)\end{array}$ & $\begin{array}{c}29 \\
(25 \mathrm{ch})\end{array}$ & $\begin{array}{c}\text { GK, dose } 10-20 \mathrm{~Gy} \\
\text { (median 15) in } 1 \text { fraction }\end{array}$ & $\sim 90 \%$ & $32 \%$ & 58 (median) & $\begin{array}{l}34 \% \mathrm{RT} \text { induced } \\
\text { complications }\end{array}$ \\
\hline $\begin{array}{l}\text { Martin et al. } \\
(2007)\end{array}$ & $\begin{array}{c}28 \\
(18 \mathrm{ch})\end{array}$ & $\begin{array}{l}\text { GK, dose: } 10.5-25 \text { Gy } \\
\text { (median 16) in } 1 \text { fraction }\end{array}$ & $63 \pm 10 \%$ & $63 \pm 10 \%$ & $\mathrm{~N} / \mathrm{A}$ & No radionecrosis \\
\hline $\begin{array}{l}\text { Hasegawa et al. } \\
(2007)\end{array}$ & $\begin{array}{c}37 \\
(30 \mathrm{ch})\end{array}$ & $\begin{array}{l}\text { GK, dose: } 9-20 \text { Gy } \\
\text { (median 14) in } 1 \text { fraction }\end{array}$ & $80 \%$ & $76 \%$ & $\begin{array}{l}\text { 10-year } \\
\text { follow-up }\end{array}$ & No radionecrosis \\
\hline $\begin{array}{l}\text { Liu et al. } \\
(2008)\end{array}$ & 31 & $\begin{array}{c}\text { GK, dose: } 10-16 \text { Gy } \\
\text { (mean 12.7) in } 1 \text { fraction }\end{array}$ & $76 \%$ & $21 \%$ & 30 (mean) & $\begin{array}{l}\text { No serious RT } \\
\text { complications }\end{array}$ \\
\hline $\begin{array}{l}\text { Dassoulas et al. } \\
\text { (2009) }\end{array}$ & 15 & $\begin{array}{l}\text { GK, dose: } 12-20 \text { Gy } \\
\text { (median 15.3) in } 1 \text { fraction }\end{array}$ & $\mathrm{N} / \mathrm{A}$ & $50 \%$ & 88 (median) & - \\
\hline $\begin{array}{l}\text { Henderson et al. } \\
\text { (2009) }\end{array}$ & 18 & $\begin{array}{l}\text { CK, dose: } 24-40 \mathrm{~Gy} \\
\text { (median 35) in } 5 \text { fractions }\end{array}$ & $\begin{array}{c}74 \% \text { (DSS: } \\
89 \%)\end{array}$ & $59 \%$ & 46 (median) & No grade 3 or 4 toxicity \\
\hline $\begin{array}{l}\text { Koga et al. } \\
(2010)\end{array}$ & $\begin{array}{c}14 \\
(10 \mathrm{ch})\end{array}$ & $\begin{array}{l}\text { GK, dose: } 10-20 \text { Gy } \\
\text { (median 15) in } 1 \text { fraction }\end{array}$ & $\mathrm{N} / \mathrm{A}$ & $43 \%$ & 65 (mean) & 14\% diplopia \\
\hline $\begin{array}{l}\text { Kano et al. } \\
(2011)\end{array}$ & 71 & $\begin{array}{c}\text { GK, dose: } 9-25 \text { Gy } \\
\text { (median 15) in } 1 \text { fraction }\end{array}$ & $80 \%$ & $66 \%$ & 60 (median) & $\begin{array}{c}9 \% \text { RT induced toxicity } \\
\text { (4\% grade } 3)\end{array}$ \\
\hline $\begin{array}{l}\text { Jiang et al. } \\
(2012)\end{array}$ & 20 & $\begin{array}{c}\text { CK, dose: } 18-50 \text { Gy } \\
\text { (mean } 32.5 \text { ) in } 1-5 \text { fractions }\end{array}$ & $53 \%$ & $55 \%$ & 34 (median) & $5 \%$ RT induced toxicity \\
\hline $\begin{array}{l}\text { Bugoci et al. } \\
(2013)\end{array}$ & 12 & $\begin{array}{c}\text { XRT, dose: } 48.6-68.4 \text { Gy } \\
\text { (median 66.6) in } 27-38 \text { fractions }\end{array}$ & $76 \%$ & $38 \%$ & 42 (median) & - \\
\hline
\end{tabular}


Table 3. Five-year overall survival and five-year progression free survival according to applied stereotactic radiosurgery technique

\begin{tabular}{|c|c|c|c|c|c|c|}
\hline Study & $\begin{array}{l}\text { Number } \\
\text { of patients }\end{array}$ & SRS/ SRT/ RT & 5-year OS & $\begin{array}{l}\text { 5-year } \\
\text { PFS }\end{array}$ & $\begin{array}{l}\text { Follow-up } \\
\text { [months] }\end{array}$ & Complications \\
\hline $\begin{array}{l}\text { Zorlu et al. } \\
\text { (2014) }\end{array}$ & 11 & $\begin{array}{l}\text { CK, dose: } 20-50 \text { Gy } \\
\text { (mean 30) in 3-5 fractions }\end{array}$ & $91 \%$ & $73 \%$ & 42 (median) & $18 \%$ radionecrosis \\
\hline $\begin{array}{l}\text { Kim et al. } \\
(2014)\end{array}$ & 5 & $\begin{array}{c}\text { GK, dose: } 13-35 \text { Gy } \\
\text { (median 20) in } 1-2 \text { fractions }\end{array}$ & $73 \%$ & $35 \%$ & 53 (mean) & 1 radionecrosis \\
\hline
\end{tabular}

ch - chordoma; CK - CyberKnife; DSS - disease specific survival; GK — Gamma Knife radiosurgery; N/A — data non available; OS — overall survival; PFS — progression free survival; RT — radiotherapy; SRS — stereotactic radiosurgery; SRT — stereotactic radiotherapy; XRT — conventional fractionated radiotherapy

Gamma Knife with higher and lower doses (18 Gy vs. 12 Gy) [36].

Radiotherapy was well tolerated and only two patients in our group experienced moderate treatment-related toxicity.

The limitations of our study are similar to those in other studies on rare tumors - a retrospective study on a small group covering a long time period, with different treatment doses and indications for irradiation. Nevertheless, due to the rarity of this tumor, studies like ours are the only way to collect the experience in this topic.

\section{Conclusion}

Our results support the conclusion that radiosurgery or hypofractionated stereotactic radiotherapy alone or in combination with surgery is a safe and effective method of irradiation of patients with skull base chordomas in any setting of its natural history which is also supported with literature data. Further studies should be performed with comparable techniques, total doses and fractionations, in a homogeneous series of patients, to obtain useful information on the role of modern radiotherapy in the treatment of chordoma.

\section{Conflict of interests}

The authors report no conflict of interest concerning the materials or methods used in this study or the findings specified in this paper.

\section{Funding}

None declared.

\section{References}

1. Zorlu F, Gultekin M, Cengiz M, et al. Fractionated stereotactic radiosurgery treatment results for skull base chordomas. Technol Cancer Res Treat. 2014; 13(1): 11-19, doi: 10.7785/tcrt.2012.500354, indexed in Pubmed: 23819495.

2. Jones PS, Aghi MK, Muzikansky A, et al. Outcomes and patterns of care in adult skull base chordomas from the Surveillance, Epidemiology, and End Results (SEER) database. J Clin Neurosci. 2014; 21(9): 1490-1496, doi: 10.1016/j.jocn.2014.02.008, indexed in Pubmed: 24852903.

3. Makhdoomi R, Ramzan A, Khursheed N, et al. Clinicopathological characteristics of chordoma: an institutional experience and a review of the literature. Turk Neurosurg. 2013; 23(6): 700-706, doi: 10.5137/1019-5149.JTN.594112.3, indexed in Pubmed: 24310451.

4. Chibbaro S, Cornelius JF, Froelich S, et al. Endoscopic endonasal approach in the management of skull base chordomas--clinical experience on a large series, technique, outcome, and pitfalls. Neurosurg Rev. 2014; 37(2): 217-24; discussion 224, doi: 10.1007/s10143-013-0503-9, indexed in Pubmed: 24249430.

5. Sen C, Triana Al, Berglind N, et al. Clival chordomas: clinical management, results, and complications in 71 patients. J Neurosurg. 2010; 113(5): 1059-1071, doi: 10.3171/2009.9.JNS08596, indexed in Pubmed: 19929198.

6. Tan NCW, Naidoo Y, Oue S, et al. Endoscopic surgery of skull base chordomas. J Neurol Surg B Skull Base. 2012; 73(6): 379-386, doi: 10.1055/s-0032-1321508, indexed in Pubmed: 24294554.

7. Rachinger W, Eigenbrod S, Dützmann S, et al. Male sex as a risk factor for the clinical course of skull base chordomas. J Neurosurg. 2014; 120(6): 13131320, doi: $10.3171 / 2013.11 . J N S 131137$, indexed in Pubmed: 24405075.

8. Amichetti M, Amelio D, Minniti G, et al. Proton therapy in chordoma of the base of the skull: a systematic review. Neurosurg Rev. 2009; 32(4): 403-416, doi: 10.1007/s10143009-0194-4, indexed in Pubmed: 19319583.

9. Fuji $\mathrm{H}$, Nakasu $Y$, Ishida $Y$, et al. Feasibility of proton beam therapy for chordoma and chondrosarcoma of the skull base. Skull Base. 2011; 21(3): 201-206, doi: 10.1055/s0031-1275636, indexed in Pubmed: 22451826.

10. Cox JD, Stetz J, Pajak TF. Toxicity criteria of the Radiation Therapy Oncology Group (RTOG) and the European Organization for Research and Treatment of Cancer (EORTC). Int J Radiat Oncol Biol Phys. 1995; 31(5): 13411346, doi: 10.1016/0360-3016(95)00060-C, indexed in Pubmed: 7713792. 
11. Almefty K, Pravdenkova S, Colli BO, et al. Chordoma and chondrosarcoma: similar, but quite different, skull base tumors. Cancer. 2007; 110(11): 2457-2467, doi: 10.1002/ cncr.23073, indexed in Pubmed: 17894390.

12. Debus J, Schulz-Ertner D, Schad L, et al. Stereotactic fractionated radiotherapy for chordomas and chondrosarcomas of the skull base. Int J Radiat Oncol Biol Phys. 2000; 47(3): 591-596, doi: 10.1016/s0360-3016(00)00464-8, indexed in Pubmed: 10837940.

13. Di Maio S, Kong E, Yip S, et al. Converging paths to progress for skull base chordoma: Review of current therapy and future molecular targets. Surg Neurol Int. 2013; 4: 72, doi: 10.4103/2152-7806.112822, indexed in Pubmed: 23776758.

14. Foweraker KL, Burton KE, Maynard SE, et al. High-dose radiotherapy in the management of chordoma and chondrosarcoma of the skull base and cervical spine: Part 1--Clinical outcomes. Clin Oncol (R Coll Radiol). 2007; 19(7): 509-516, doi: 10.1016/j.clon.2007.04.004, indexed in Pubmed: 17524633.

15. Hug EB, Loredo LN, Slater JD, et al. Proton radiation therapy for chordomas and chondrosarcomas of the skull base. J Neurosurg. 1999; 91(3): 432-439, doi: 10.3171/ jns.1999.91.3.0432, indexed in Pubmed: 10470818.

16. Igaki $H$, Tokuuye $K$, Okumura $T$, et al. Clinical results of proton beam therapy for skull base chordoma. Int J Radiat Oncol Biol Phys. 2004; 60(4): 1120-1126, doi: 10.1016/j. ijrobp.2004.05.064, indexed in Pubmed: 15519783.

17. Ito $E$, Saito $K$, Okada T, et al. Long-term control of clival chordoma with initial aggressive surgical resection and gamma knife radiosurgery for recurrence. Acta Neurochir (Wien). 2010; 152(1): 57-67; discussion 67, doi: 10.1007/ s00701-009-0535-7, indexed in Pubmed: 19826755.

18. Noël G, Feuvret L, Calugaru V, et al. Chordomas of the base of the skull and upper cervical spine. One hundred patients irradiated by a 3D conformal technique combining photon and proton beams. Acta Oncol. 2005; 44(7): 700-708, doi: 10.1080/02841860500326257, indexed in Pubmed: 16227160.

19. Pallini R, Maira G, Pierconti F, et al. Chordoma of the skull base: predictors of tumor recurrence. J Neurosurg. 2003; 98(4): 812-822, doi: 10.3171/jns.2003.98.4.0812, indexed in Pubmed: 12691407.

20. Pamir MN, Ozduman K. Tumor-biology and current treatment of skull-base chordomas. Adv Tech Stand Neurosurg. 2008; 33: 35-129, doi: 10.1007/978-3-211-72283-1_2, indexed in Pubmed: 18383812.

21. Samii A, Gerganov VM, Herold C, et al. Chordomas of the skull base: surgical management and outcome. J Neurosurg. 2007; 107(2):319-324, doi: 10.3171/JNS-07/08/0319, indexed in Pubmed: 17695386.

22. Schulz-Ertner D, Karger CP, Feuerhake A, et al. Effectiveness of carbon ion radiotherapy in the treatment of skull-base chordomas. Int J Radiat Oncol Biol Phys. 2007; 68(2):449-457, doi: 10.1016/j.jirobp.2006.12.059, indexed in Pubmed: 17363188.

23. Stüer C, Schramm J, Schaller C. Skull base chordomas: management and results. Neurol Med Chir (Tokyo). 2006; 46(3): 118-24; discussion 124, doi: 10.2176/nmc.46.118, indexed in Pubmed: 16565581.

24. Takahashi S, Kawase T, Yoshida K, et al. Skull base chordomas: efficacy of surgery followed by carbon ion radiotherapy. Acta Neurochir (Wien). 2009; 151(7):
759-769, doi: 10.1007/s00701-009-0383-5, indexed in Pubmed: 19434365.

25. Tamaki N, Nagashima T, Ehara K, et al. Surgical approaches and strategies for skull base chordomas. Neurosurg Focus. 2001; 10(3): E9, doi: 10.3171/foc.2001.10.3.10, indexed in Pubmed: 16734412.

26. Tzortzidis F, Elahi F, Wright D, et al. Patient outcome at long-term follow-up after aggressive microsurgical resection of cranial base chordomas. Neurosurgery. 2006; 59(2): 230-7; discussion 230, doi: 10.1227/01.NEU.0000223441.51012.9D, indexed in Pubmed: 16883163.

27. Yoneoka Y, Tsumanuma I, Fukuda M, et al. Cranial base chordoma--long term outcome and review of the literature. Acta Neurochir (Wien). 2008; 150(8): 773-8; discussion 778, doi: 10.1007/s00701-008-1600-3, indexed in Pubmed: 18548191.

28. Bugoci DM, Girvigian MR, Chen JCT, et al. Photon-based fractionated stereotactic radiotherapy for postoperative treatment of skull base chordomas. Am J Clin Oncol. 2013; 36(4): 404-410, doi: 10.1097/COC.0b013e318248dc6f, indexed in Pubmed: 22772429.

29. Chang SD, Martin DP, Lee E, et al. Stereotactic radiosurgery and hypofractionated stereotactic radiotherapy for residual or recurrent cranial base and cervical chordomas. Neurosurg Focus. 2001; 10(3): E5, doi: 10.3171/ foc.2001.10.3.6, indexed in Pubmed: 16734408.

30. Dassoulas K, Schlesinger D, Yen CPo, et al. The role of Gamma Knife surgery in the treatment of skull base chordomas. J Neurooncol. 2009; 94(2): 243-248, doi: 10.1007/s11060-009-9846-z, indexed in Pubmed: 19277468.

31. Hasegawa T, Ishii D, Kida Y, et al. Gamma Knife surgery for skull base chordomas and chondrosarcomas. J Neurosurg. 2007; 107(4): 752-757, doi: 10.3171/JNS-07/10/0752, indexed in Pubmed: 17937219.

32. Henderson FC, McCool K, Seigle J, et al. Treatment of chordomas with Cyberknife: georgetown university experience and treatment recommendations. Neurosurgery. 2009; 64(2 Suppl): A44-A53, doi: 10.1227/01.NEU.0000341166.09107.47, indexed in Pubmed: 19165073.

33. Jiang $B$, Veeravagu $A$, Lee $M$, et al. Management of intracranial and extracranial chordomas with CyberKnife stereotactic radiosurgery. J Clin Neurosci. 2012; 19(8): 1101-1106, doi: 10.1016/j.jocn.2012.01.005, indexed in Pubmed: 22727205.

34. Kano H, Iqbal FO, Sheehan J, et al. Stereotactic radiosurgery for chordoma: a report from the North American Gamma Knife Consortium. Neurosurgery. 2011; 68(2): 379-389, doi: 10.1227/NEU.0b013e3181ffa12c, indexed in Pubmed: 21135744

35. Kim JiH, Jung HHo, Chang JH, et al. Gamma Knife surgery for intracranial chordoma and chondrosarcoma: radiosurgical perspectives and treatment outcomes. J Neurosurg. 2014; 121 Suppl: 188-197, doi: 10.3171/2014.7.GKS141213, indexed in Pubmed: 25434952.

36. Koga T, Shin M, Saito N. Treatment with high marginal dose is mandatory to achieve long-term control of skull base chordomas and chondrosarcomas by means of stereotactic radiosurgery. J Neurooncol. 2010; 98(2): 233-238, doi: 10.1007/s11060-010-0184-y, indexed in Pubmed: 20419388. 
37. Krishnan S, Foote RL, Brown PD, et al. Radiosurgery for cranial base chordomas and chondrosarcomas. Neurosurgery. 2005; 56(4): 777-84; discussion 777, doi: 10.1227/01. neu.0000156789.10394.f5, indexed in Pubmed: 15792516.

38. Liu AL, Wang ZC, Sun SB, et al. Gamma knife radiosurgery for residual skull base chordomas. Neurol Res. 2008; 30(6): 557-561, doi: 10.1179/174313208X297878, indexed in Pubmed: 18647493.
39. Martin JJ, Niranjan A, Kondziolka D, et al. Radiosurgery for chordomas and chondrosarcomas of the skull base. J Neurosurg. 2007; 107(4): 758-764, doi: 10.3171/JNS07/10/0758, indexed in Pubmed: 17937220.

40. Muthukumar N, Kondziolka D, Lunsford LD, et al. Stereotactic radiosurgery for chordoma and chondrosarcoma: further experiences. Int J Radiat Oncol Biol Phys. 1998; 41(2): 387-392, doi: 10.1016/s0360-3016(98)00051-0, indexed in Pubmed: 9607355. 